\title{
Antimicrobial susceptibility pattern for Enterococcus species colonizing the GIT of hospitalized and community patients
}

\author{
Ramamoorthi Arularasi Aberna ${ }^{1, *}$, Kesani Prabhakar ${ }^{2}$ \\ ${ }^{1}$ Lecturer, Dept. of Microbiology, Rajah Muthiah Dental College \& Hospital Annamalai University, Tamil Nadu, \\ ${ }^{2}$ Professor, Dept. of Microbiology, Rajah Muthiah Medical College \& Hospital Annamalai University, Tamil Nadu, India
}

*Corresponding Author:

Email: arularasiaberna@gmail.com

Received: $6^{\text {th }}$ April, 2018

Accepted: $15^{\text {th }}$ May, 2018

\begin{abstract}
Objective: To determine the species of Enterococcus prevalent in the gastrointestinal tract (GIT) of patients in hospitals and community and to determine the antimicrobial susceptibility pattern for the isolates.

Materials and Methods: Enterococcus species were isolated from stools and rectal swabs using selective media. Antimicrobial susceptibility pattern for 9 antibiotics were evaluated by Kirby-Bauer's disc diffusion method. Phenotypic characterization of vancomycin resistant enterococcus (VRE) isolate was done by determining the minimum inhibitory concentration (MIC) to vancomycin and teicoplanin by agar dilution method.

Results: Enterococcus species were isolated from gastrointestinal tracts among $74.4 \%$ of hospitalized patients and $90 \%$ of community patients. E. faecium was the frequent species isolated from both patients. Hospitalized patients had lesser number of other unusual Enterococcus species. Resistance to antibiotics was higher among isolates from hospitalized patients and $1.49 \%$ of hospitalized patients showed colonization with VanB phenotype VRE.

Conclusion: Gastrointestinal carriage of antibiotic resistant enterococci including VRE among hospitalized patients mandates the need for periodic surveillance among high risk patients in order to prevent infections and dissemination.
\end{abstract}

Keywords: Gastrointestinal carriage, Enterococcus, Antibiotic resistance, VRE, Hospitalized and community patients.

\section{Introduction}

Enterococci species have gained significance as leading cause of hospital acquired bacteraemia, endocarditis and urinary tract infections. Of more than a fifty species in the genus, E. faecalis followed by $E$ faecium accounts for $80-90 \%$ and $10-15 \%$ of clinical infections respectively. ${ }^{1-3}$ Infections by other unusual species including E. casseliflavus, E. mundtii, $E$. gallinarum, E. durans, E. dispar and E. durans are being reported from clinical specimens. ${ }^{4}$ Enterococcal infections are a significant cause of concern because of their ability to resist wide range of antimicrobials by intrinsic and extrinsic resistance mechanisms. ${ }^{5}$ Multiple drug resistant isolates contribute to increased length of hospitalisation, co-morbidities, increased mortality rates and inter and intra hospital spread. ${ }^{6}$

Enterococci are commensals of gastrointestinal tract and majority of the infections have found to be evolved from the patient's own flora. The selective pressure due to antibiotic administration perturbs the symbiotic relationship of GIT commensals resulting in proliferation of drug resistant isolates which then translocates to cause severe systemic infections. ${ }^{6,7}$ Colonisation by vancomycin resistant enterococci and aminoglycoside resistant enterococci in the GIT are reported with the risk of developing blood stream infections and endocarditis. Hence, recognition of asymptomatic gastrointestinal reservoirs of antibiotic resistant Enterococcus isolates could be vital to prevent subsequent infections and dissemination leading to epidemics. ${ }^{1,8}$
Considering the dual role of enterococci as commensals and nosocomial pathogens, a study was aimed to investigate the presence of antibiotic resistance in intestinal colonizers among hospitalized patients and community patients. The findings of the study will help to generate epidemiological data in our geographical area, address the risk related to systemic infections besides insinuating the need for periodic surveillance to circumvent strain dissemination.

\section{Materials and Methods}

The study was carried out in patients attending services of the Rajah Muthiah Medical College and Hospital, Annamalai University from June 2016 to December 2016. The study included faecal samples or rectal swabs obtained from 90 hospitalised patients and 60 community patients.

Inclusion and Exclusion Criteria: Hospitalized patients: Patients admitted to ICU, general medicine, paediatrics, OG and surgery wards with more than 5 days of hospitalisation were enrolled for the study. Community patients: Patients attending the OPD with or without diarrhoea, no history of prior hospitalisation for past 6 months and were not on antibiotic therapy for past 6 months were included in the study.

Specimen Collection: Samples were collected after obtaining informed consent from the patient or attendant. Stool specimens were collected in sterile, plastic wide mouth containers. The samples were transported to the Department of Microbiology, within 30 minutes and processed further. Rectal swabs were 
obtained in sterile cotton swabs moistened with sterile saline. The swabs were transported in Cary-Blair transport media to laboratory within 30 minutes.

Culture and Identification: Rectal swabs and stool samples were inoculated on bile esculin agar and Pfizer selective enterococcus agar (Hi-Media Laboratories, India) and incubated at $37^{\circ} \mathrm{C}$ for overnight period. Colonies with dark brown halo and morphologically resembling enterococci were characterized by conventional test scheme including by Grams stain, catalase test, heat tolerance test, and arginine hydrolysis, ability to grow at $45^{\circ} \mathrm{C}$ and in the presence of $6.5 \% \mathrm{NaCl}$. Species identification of enterococci included test for pigment production, motility, efrotomycin (EFRO) test (Hi-Media Laboratories, India), acidification of MGP (1-)-methyl-alpha Dglucopyranoside) and sugar fermentation tests as recommended earlier.9,10 Carbohydrate fermentation tests were done in Phenol red broth base medium containing $1 \%$ of carbohydrate-mannitol, sucrose, sorbitol, raffinose, pyruvate, arabinose, trehalose, lactose, xylose and ribose.

Antimicrobial Susceptibility Testing: The ability of antibiotics to inhibit the growth of gastrointestinal isolates of Enterococci was evaluated by Kirby-Bauer's disc diffusion method on Mueller Hinton agar using $E$. faecalis ATCC 29212 as quality control strain. The antibiotic discs included were penicillin [10 units], ampicillin $[10 \mu \mathrm{g}]$, gentamicin-high content [120 $\mu \mathrm{g}$ ], streptomycin-high content $[300 \mu \mathrm{g}]$, erythromycin [15 $\mu \mathrm{g}$, ciprofloxacin [5 $\mu \mathrm{g}]$, vancomycin $\left[\begin{array}{ll}30 & \mu \mathrm{g}\end{array}\right]$, teicoplanin $[30 \mu \mathrm{g}]$ and linezolid $[30 \mu \mathrm{g}]$. The discs were purchased from Hi-Media Laboratories, India and stored at $4^{\circ} \mathrm{C}$ until use. The test was performed and the results were interpreted according to CLSI, 2012. ${ }^{11}$

Phenotypic Characterization of Vancomycin Resistance: Phenotypic characterization of vancomycin resistance was based on the minimum inhibitory concentration (MIC) of the isolates to vancomycin and teicoplanin antibiotics by agar dilution method. MIC was determined according to CLSI, 2012. ${ }^{11}$ VanA phenotype was characterized by MIC value $\geq 64 \mu \mathrm{g} / \mathrm{mL}$ for vancomycin and $\geq 16 \mu \mathrm{g} / \mathrm{mL}$ for teicoplanin. Isolates with MIC value of $8-64 \mu \mathrm{g} / \mathrm{mL}$ for vancomycin, $\leq 1$ $\mu \mathrm{g} / \mathrm{mL}$ for teicoplanin were characterized as VanB phenotype.

\section{Statistical Analysis}

Fisher exact test was applied to determine the significance of difference between the resistance levels using SPSS version 15. $\mathrm{P} \leq 0.01$ was considered as significant.

\section{Results}

Isolation Rate of Enterococci: Gastrointestinal carriage of enterococci was screened among 150 hospitalized and community patients. One hundred and twenty one Enterococcus isolates were derived from stool and rectal swabs. Enterococcus was isolated from 67 of hospitalized patients and 54of patients living in community.

Distribution of Enterococcus Species among the Gastrointestinal Carriers: E. faecalis and E. faecium were isolated from $41.8 \%$ and $59.6 \%$ of the hospitalized patients and $35.2 \%$ and $37 \%$ of the community derived patients respectively. Five species of unusual Enterococcus species were isolated from the study groups. The prevalence of E. avium and E. raffinosus were observed only among the patients from the community (Table 1), (Fig. $1-4)$.

Antimicrobial Resistance Pattern of Gastrointestinal Isolates: Isolates from hospitalized patients demonstrated a higher percentage of resistance compared to the community patients (Fig 5). Resistance towards penicillin was found in $37 \%$ of isolates from both sources. Two vancomycin resistant enterococci (VRE) were isolated from hospitalized patients (Fig. 6).

VRE Colonisation: One VRE isolate was obtained from stool sample of a male, hospitalized patient aged 67 , with history of diabetes, recent abdominal surgery, catheterization, intake of $3^{\text {rd }}$ generation cephalosporin antibiotic and hospitalization for more than 10 days. The isolate was identified as E. faecium and was found resistant to penicillin, ampicillin, gentamicin, ciprofloxacin and linezolid. Phenotypic characterization of the VRE isolate revealed an MIC for vancomycin and teicoplanin as $64 \mu \mathrm{g} / \mathrm{mL}$ and $1 \mu \mathrm{g} / \mathrm{mL}$ respectively and hence belonged to VanB phenotype (Fig. 7, 8).

Table 1: Distribution of Enterococcus species among the gastrointestinal carriers:

\begin{tabular}{|l|c|c|c|c|c|c|}
\hline \multirow{2}{*}{$\begin{array}{c}\text { Enterococcus } \\
\text { species }\end{array}$} & $\begin{array}{c}\text { Hospitalized patients } \\
(\mathbf{n}=\mathbf{6 7})\end{array}$ & \multicolumn{2}{c|}{$\begin{array}{c}\text { Community patients } \\
(\mathbf{n}=\mathbf{5 4})\end{array}$} & \multicolumn{2}{c|}{$\begin{array}{c}\text { Total } \\
(\mathbf{n = 1 2 1})\end{array}$} \\
\cline { 2 - 7 } & $\begin{array}{c}\text { No. of } \\
\text { isolates }\end{array}$ & Percentage & No. of isolates & Percentage & No. of isolates & Percentage \\
\hline E. faecium & 31 & 59.6 & 20 & 37 & 51 & 42.1 \\
\hline E. faecalis & 28 & 41.8 & 19 & 35.2 & 47 & 38.8 \\
\hline E. gallinarum & 2 & 3 & 7 & 13 & 9 & 7.4 \\
\hline E. casseliflavus & 4 & 6 & 2 & 3.7 & 6 & 5 \\
\hline E. raffinosus & 0 & 0 & 2 & 3.7 & 2 & 1.7 \\
\hline E. avium & 0 & 0 & 2 & 3.7 & 2 & 1.7 \\
\hline E. hirae & 2 & 2.9 & 2 & 3.7 & 4 & 3.3 \\
\hline
\end{tabular}




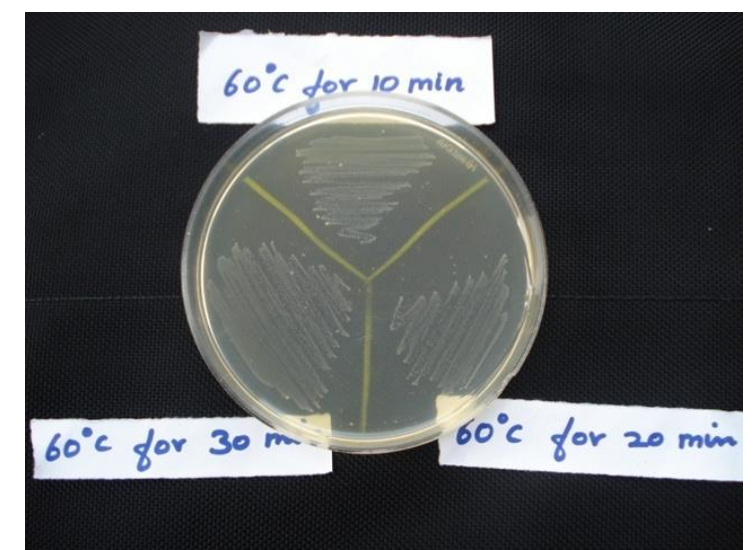

Fig. 1: Heat tolerance test for Enterococcus identification

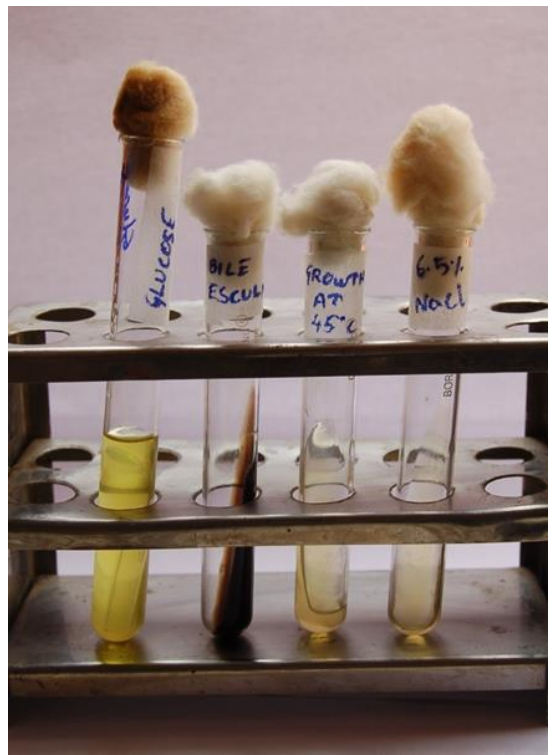

Fig. 2: Tests for Enterococcus identification

Glucose fermentation, Bile esculin + , Growth at $45^{\circ} \mathrm{C}$ and in the presence of $6.5 \% \mathrm{NaCl}$

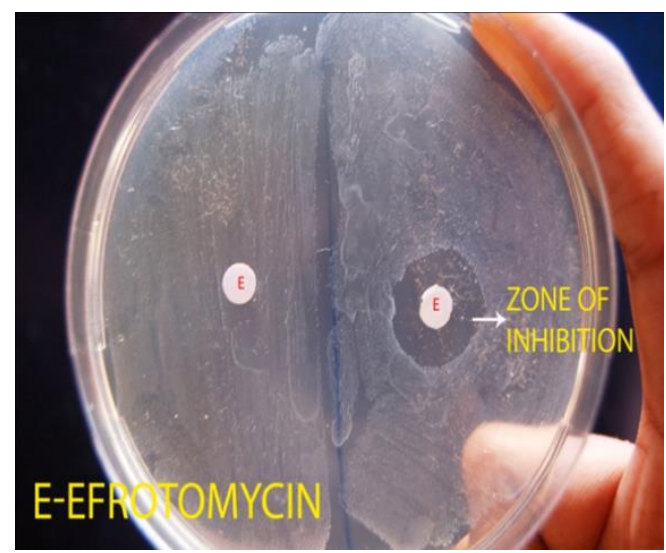

Fig. 3: Efro test

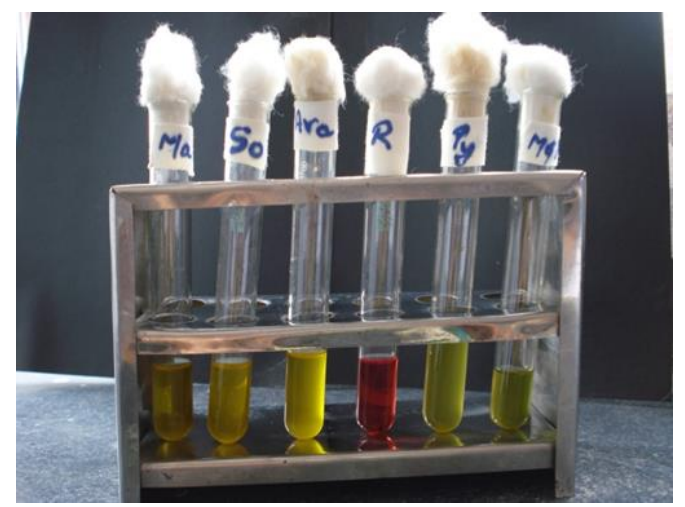

Fig. 4: Carbohydrate fermentation tests

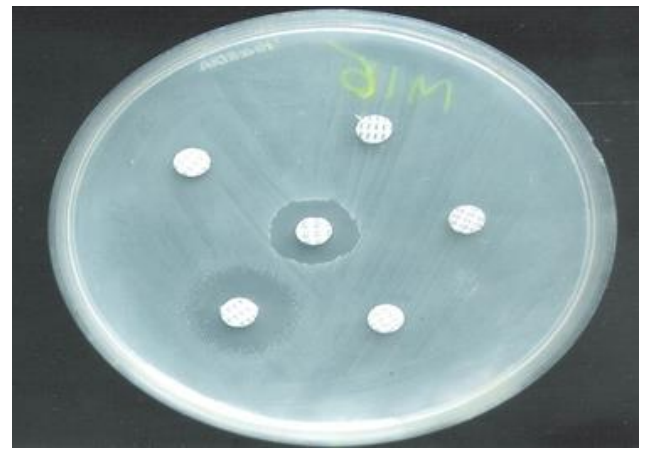

Fig. 5: Disc diffusion test

Presence and absence of zone of inhibition around the antibiotic discs

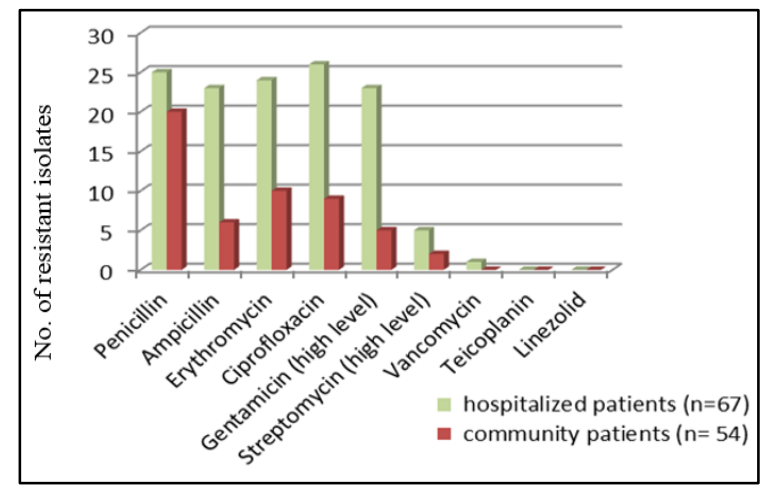

Fig. 6: Antimicrobial resistance pattern of gastrointestinal isolates

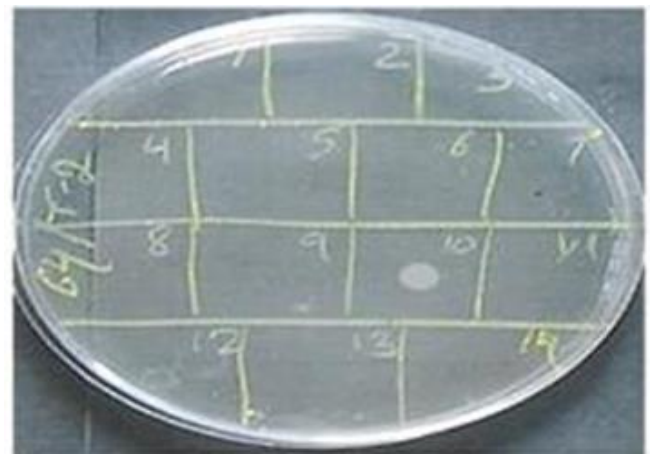

Fig. 7: Phenotypic characterization by agar dilution for vancomycin 


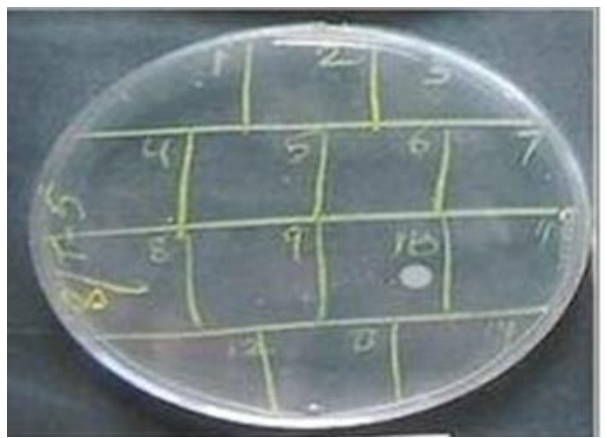

Fig. 8: Phenotypic characterization by agar dilution for teicoplanin

\section{Discussion}

The gastrointestinal tract exhibiting resistant enterococci acts as reservoir of infections. ${ }^{3}$ In our study enterococci were isolated from $74.4 \%$ of hospitalized patients and $90 \%$ of community patients. Previous investigations of stool samples from hospitalized patients showed the prevalence of enterococci around $71.2 \%$ - 77\%..$^{12,13}$ Among community patients the GIT colonization of enterococci ranged from $85.5 \%$ $100 \% .^{14,15}$ Similar observations in our study supports the fact that usage of broad spectrum antimicrobials in the hospital may have altered the GIT flora leading to their lower isolation rates. ${ }^{16}$

E. faecium was the frequently isolated species from both hospitalized and community patients however, its isolation percentage was higher among hospitalised patients $(59.6 \%)$ compared to community patients (38.9\%). Reports on isolation of E. faecium ranging from $60.5 \%-71 \%$ among hospitalized patients and $29 \%$ $39.6 \%$ among community patients by other workers is in accordance with our study. ${ }^{13,17}$ It could be comprehended that the intrinsic nature of drug resistance associated with E. faecium compared to other species could have offered a selective advantage to emerge in higher numbers. ${ }^{1}$ The unusual Enterococcus species frequently isolated from stool samples are $E$. avium, E. raffinosus, E.durans, E. mundtii and E.casseliflavus. ${ }^{12,13,17}$ In our study three and five species of unusual enterococci were obtained from hospitalized and community patients respectively. The isolation of lesser number of unusual Enterococcus species among hospitalized patients is a clear indication of the role of antibiotics against other Enterococcus species which are well known for their heightened susceptibility to antibiotics. ${ }^{1}$

Resistance rate towards penicillin antibiotic was found to be same (37\%) among isolates from both sources. Similar resistance rates for penicillin among isolates from both sources could be attributed to the frequent use of penicillin in the community for the empirical treatment of infectious diseases. Enterococci possess an intrinsic resistance (chromosomally coded) to betalactum antibiotics by producing penicillin- binding protein 5 (PBP5) which has low affinity to the drug and continues peptidoglycan synthesis. ${ }^{3}$

The resistance percentage to ampicillin, erythromycin, ciprofloxacin and gentamicin antibiotics were higher among the isolates from hospitalized patients and this increased levels was found statistically significant $(\mathrm{P} \leq 0.01)$ compared to community derived isolates. Resistance to high level gentamicin is by production of aminoglycoside modifying enzymes whose action eliminates the possibility of synergistic treatment of enterococcal infections with cell wall inhibiting agent-ampicillin or vancomycin. ${ }^{18}$ Considering the characteristic nature of enterococci to survive in harsh environment outside the GIT and its possibility to be transmitted via health care workers, the GIT colonization by drug resistant Enterococcus among hospitalized patients raises serious concern in our setting. ${ }^{19}$

VRE colonization was observed in $1.49 \%$ of hospitalized patients in our study. Investigations on VRE colonization from Belgium, UK, Netherland and Finland have reported a prevalence of 2-3.5\% among hospitalized patients. ${ }^{21-22}$ The risk factors for VRE colonization as described by Safdar et al., 2002 including long term hospitalization, surgery, catheterization, exposure to $3^{\text {rd }}$ generation cephalosporin correlates well with the history of VRE colonized patient in our study. ${ }^{23}$ This signals the need for surveillance among high risk groups to implement infection containment measures.

\section{Conclusion}

Gastrointestinal carriage of antibiotic resistant Enterococcus species was marked among the hospitalized patients compared to community counterparts. This proves the role of selective pressure of antibiotics in altering the GIT flora which may precipitate serious clinical infections. Occurrence of VRE isolate and existence of increased number of isolates expressing resistance to antibiotics used for synergistic treatment mandates measures for surveillance at least among high risk groups. The study coerces the need for stringent infection control procedures in order to prevent infection and dissemination of resistant isolates colonizing intestinal tract of hospitalized patients.

\section{References}

1. Sood S, Das MM, Kapil A. Enterococcal infections and antimicrobial resistance. Indian J Med Res. 2008;128:111-121.

2. Udo E, Al-Sweih N, John P, Chugh TD. Antibiotic resistance of enterococci isolated at a teaching hospital in Kuwait. Diag Microbiol Infect Dis. 2002;43:233-238.

3. Dubin K, Pamer EG. Enterococci and their interactions with the intestinal microbiome. Microbiology spectrum. 2014;5(6).

4. Prakash VP, Rao SR, Parija SC. Emergence of unusual species of enterococci causing infections, South India. BMC infectious diseases. 2005;5(1):14. 
5. Miller WR, Munita JM, Arias CA. Mechanisms of antibiotic resistance in enterococci. Expert review of antiinfective therapy. 2014;12(10):1221-36.

6. Papadimitriou-Olivgeris M, Drougka E, Fligou F, Kolonitsiou F, Liakopoulos A, Dodou V, Anastassiou ED, Petinaki E, Marangos M, Filos KS, Spiliopoulou I. Risk factors for enterococcal infection and colonization by vancomycin-resistant enterococci in critically ill patients. Infection. 2014;42(6):1013-22.

7. Salyers AA, Gupta A, Wang Y. Human intestinal bacteria as reservoirs for antibiotic resistance genes. Trends in microbiology. 2004;12(9):412-6.

8. Kara A, Devrim I, Bayram N, Katipoglu N, Kıran E, Oruc Y, Demiray N, Apab H, Gulfidan G. Risk of vancomycin-resistant enterococci bloodstream infection among patients colonized with vancomycin-resistant enterococci. Brazilian Journal of Infectious Diseases. 2015;19(1):58-61.

9. Facklam RR, Sahm DF, Texeira LM: Enterococcus. Manual of clinical microbiology. Edited by: Murray PR, Baron EJ, Pfaller MA, Tenover FC, Yolken RH. 1999, ASM press, Washington, DC, 297-305.

10. Facklam RR, Collins MD: Identification of Enterococcus species isolated from human infections by a conventional test scheme. J Clin Microbiol. 1989;27:731-4.

11. CLSI. 2012. Methods for dilution antimicrobial susceptibility tests for bacteria that grow aerobically; approved standard, 9th ed. CLSI document M07-A9. CLSI, Wayne, PA.

12. Moaddab SR, Rafi A. Prevalence of vancomycin and high level aminoglycoside resistant enterococci among highrisk patients. Southeast Asian J Trop Med Public Health. 2003;34(4):849-54.

13. Yildirim M, Sencan I, Ozdemir D, Oksuz S, Yilmaz Z, Sahin I. Vancomycin and high-level aminoglycoside resistant Enterococcus carriage and the risk factors related to resistance in hospitalized patients. Mikrobiyoloji bulteni. 2007;41(2):271-7.

14. Barreto A, Guimaraes B, Radhouani H, Araujo C, Goncalves A, Gaspar E, Rodrigues J, Igrejas G, Poeta P. Detection of antibiotic resistant E. coli and Enterococcus spp. in stool of healthy growing children in Portugal. Journal of basic microbiology. 2009;49(6):503-12.

15. Coque TM, Tomayko JF, Ricke SC, Okhyusen PC, Murray BE. Vancomycin-resistant enterococci from nosocomial, community, and animal sources in the United States. Antimicrobial agents and chemotherapy. 1996;40(11):2605-9.

16. Pegues DA, Pegues CF, Hibberd PL, Ford DS, Hooper DC. Emergence and dissemination of a highly vancomycin-resistant vanA strain of Enterococcus faecium at a large teaching hospital. Journal of clinical microbiology. 1997;35(6):1565-70.

17. Sekar R, Srivani R, Vignesh R, Kownhar H, Shankar EM. Low recovery rates of high-level aminoglycosideresistant enterococci could be attributable to restricted usage of aminoglycosides in Indian settings. Journal of medical microbiology. 2008;1;57(3):397-8.

18. Padmasini E, Padmaraj R, Ramesh SS. High level aminoglycoside resistance and distribution of aminoglycoside resistant genes among clinical isolates of Enterococcus species in Chennai, India. The Scientific World Journal. 2014;2014.

19. Duckro AN, Blom DW, Lyle EA, Weinstein RA, Hayden MK. Transfer of vancomycin-resistant enterococci via health care worker hands. Archives of internal medicine. 2005;165(3):302-7.

20. Endtz HP, van den Braak N, van Belkum A, Kluytmans JA, Koeleman JG, Spanjaard L, et al,. Fecal carriage of vancomycin-resistant enterococci in hospitalized patients and those living in the community in The Netherlands. Journal of Clinical Microbiology. 1997;35(12):3026-31.

21. Gordts B, Van Landuyt HE, Leven M, Vandamme P, Goossens H. Vancomycin-resistant enterococci colonizing the intestinal tracts of hospitalized patients. Journal of Clinical Microbiology. 1995;33(11):2842-6.

22. Suppola JP, Volin L, Valtonen VV, Vaara M. Overgrowth of Enterococcus faecium in the feces of patients with hematologic malignancies. Clinical infectious diseases. 1996;23(4):694-7.

23. Safdar N, Maki DG. The commonality of risk factors for nosocomial colonization and infection with antimicrobialresistant Staphylococcus aureus, enterococcus, gramnegative bacilli, Clostridium difficile, and Candida. Annals of Internal Medicine. 2002;136(11):834-44.

How to cite this article: Aberna RA, Prabhakar K. Antimicrobial susceptibility pattern for Enterococcus species colonizing the GIT of hospitalized and community patients. Indian J Microbiol Res. 2018;5(3):290-294. 